\title{
Le Chemin des correspondances et le champ poétique. À la mémoire de Michael Pakenham, dir. de S. Murphy
}

\section{Maria Emanuela Raffi}

\section{(2) OpenEdition}

1 Journals

\section{Edizione digitale}

URL: http://journals.openedition.org/studifrancesi/10699

DOI: 10.4000/studifrancesi.10699

ISSN: 2421-5856

\section{Editore}

Rosenberg \& Sellier

\section{Edizione cartacea}

Data di pubblicazione: 1 décembre 2017

Paginazione: $573-577$

ISSN: 0039-2944

\section{Notizia bibliografica digitale}

Maria Emanuela Raffi, «Le Chemin des correspondances et le champ poétique. À la mémoire de Michael Pakenham, dir. de S. Murphy», Studi Francesi [Online], 183 (LXI | III) | 2017, online dal 01 février 2018, consultato il 22 janvier 2021. URL: http://journals.openedition.org/studifrancesi/10699 ; DOI: https:// doi.org/10.4000/studifrancesi.10699

Questo documento è stato generato automaticamente il 22 janvier 2021.

\section{(c) 9 (i) $\Theta$}

Studi Francesi è distribuita con Licenza Creative Commons Attribuzione - Non commerciale - Non opere derivate 4.0 Internazionale. 


\title{
Le Chemin des correspondances et le champ poétique. À la mémoire de Michael Pakenham, dir. de S. Murphy
}

\author{
Maria Emanuela Raffi
}

\section{NOTIZIA}

Le Chemin des correspondances et le champ poétique. À la mémoire de Michael Pakenham, sous la direction de Steve MURPHY, Paris, Classiques Garnier, 2016, 705 pp.

1 Il considerevole numero di contributi che formano questo volume sottolinea anzitutto l'importanza dello studioso, Michael Pakenham, cui vogliono rendere omaggio, ma anche la fitta rete di legami scientifici e affettivi intessuti nella sua ricca esistenza.

2 È Steve MURPHY a tratteggiarne il ritratto di studioso e ricercatore, appassionato di grandi poeti - Baudelaire, Verlaine, Rimbaud, Mallarmé - ma anche di «mineurs», come Pradelle, Jules Mary, Louis-Xavier de Ricard, Mérat e soprattutto Nouveau. L'immagine di Pakenham «homme d'amitié» e generoso collaboratore in pubblicazioni collettive e in riviste scientifiche completa il ritratto, il cui ultimo importante elemento è rappresentato dall'edizione e l'annotazione della corrispondenza di Verlaine, lavoro impervio per i numerosi controlli necessari sulle edizioni precedenti, la difficile datazione di molte lettere, la presenza di molti inediti e l'infinita pazienza richiesta per la realizzazione dei tre volumi, il primo pubblicato nel 2005, il secondo in via di pubblicazione e il terzo affidato all'équipe di Fayard sulla base della documentazione in gran parte già raccolta da Pakenham.

Il ricordo scritto da Keith CAMERon, frutto di una frequentazione di quasi mezzo secolo, produce un vivace ritratto di Michael Pakenham, della sua vastissima cultura e della sua personalità «ayant des facettes à la fois modestes et flamboyantes».

4 Luce ABÉLÈs, amica e collaboratrice di Pakenham nell'allestimento della mostra La Dame aux éventails. Nina de Callias, modèle de Manet, dedica il suo contributo («Je suis commerçant 
mais un peu artiste aussi») a Léon Vanier, editore dell'opera di Verlaine nonché direttore di alcuni giornali illustrati, partendo da due lettere inedite scritte da Vanier all'attore Mévisto. L'A. offre un'approfondita presentazione di «Les Hommes d'aujourd'hui», cui ha collaborato lungamente Verlaine, esaminandone i due aspetti fondamentali: le illustrazioni di copertina e le biografie di artisti e scrittori celebri e di altri più innovatori e meno noti, secondo la definizione di sé data dallo stesso Vanier: «commerçant, mais un peu artiste aussi».

In Répertoire du dixain réaliste, Jean-Louis AROUI presenta «l'état actuel» dell'ambizioso progetto di pubblicare un repertorio di tutti i dixains réalistes esistenti. Vengono quindi esposti da Aroui i criteri formali fondamentali di inclusione - serie di poesie formate da "1 dixain d'alexandrins à rimes plates», poesie con un multiplo di 10 versi in cui il dixain funziona come una strofa, serie di dixains con qualche variante che non ne snatura il genere ecc. - e l'indicazione dei 59 gruppi in cui sono suddivisi i 759 dixains réalistes finora reperiti.

6 Il poeta Jean Aicard, collaboratore della «Renaissance littéraire et artistique» ben noto a Michael Pakenham, è l'oggetto dello studio di Leisha ASHDOWN-LECOINTRE, Lettres et l'être du poète. Basandosi sulla corrispondenza di Aicard, l'A. mette in rilievo anzitutto il forte legame con Victor Hugo, prima sostenitore convinto del quasi ignoto Aicard, poi a sua volta oggetto delle recensioni dell'allievo, di cui sostiene la grandezza per tutta la vita. Molto importanti appaiono, per un autore che oscilla geograficamente ed emotivamente fra la Provenza e Parigi, anche i rapporti con gli altri poeti provenzali come Autran e Victor de Laprade, ma anche l'approvazione di autori affermati, soprattutto quella di George Sand.

7 Christophe BATAILLÉ propone alcune interessanti riflessioni su un testo fra i meno frequentati di Rimbaud: "Le Rêve de Bismarck" de Rimbaud ou le fin mot de l'histoire. Benché pubblicato, in prosa, nel «Progrès des Ardennes» nel 1870 da un giovanissimo Rimbaud, il Rêve si rivela per Bataillé "plus subtil qu'il n'y paraît», grazie soprattutto al lavoro sulla lingua: un gioco di rimandi fra senso proprio e senso figurato, l'utilizzo «des expressions lexicalisées», la rielaborazione di citazioni (Tartuffe) e infine i doppi sensi di carattere erotico e le metafore oscene, che legano strettamente Le Rêve de Bismarck al suo contemporaneo Un cour sous une soutane.

8 Spostando l'attenzione su un altro autore caro a Pakenham, Arnaud BERNADET ("Sagesse", la religion d'un maniaque) illustra le ragioni dell'accoglienza piuttosto ostile riservata a Sagesse di Verlaine, come anche a Romances sans parole, e del rovesciamento del giudizio su queste opere «qui se révéleront capitales par la suite» in ambito simbolistadecadente. Partendo dall'interpretazione di Henry Céard, recuperata da Pakenham, Bernardet legge la religione di Sagesse come espressione di una patologia maniacale, cosa che permette sia di collegare questa raccolta con le precedenti opere non religiose e con l'esperienza giudiziaria nel carcere di Bruxelles, sia di leggerla come prodotto della «pathologie artistique» di derivazione baudelairiana, profondamente sospetta agli occhi dei critici cattolici.

9 Patrick BESNIER rende conto nel suo contributo, En hommage à Sylvain Itkine, della serata in onore dell'attore e regista Itkine realizzata al teatro Récamier nel giugno del $1946 \mathrm{da}$ Michel de Ré, composta in gran parte da opere di Jarry, con un testo di Chrétien de Troyes, uno di Ribemont-Desseignes, uno di Michaux, uno di Hernando Ruiz de Alarcón e infine La Chanson de la faim dello stesso Itkine e di Maurice Marc. 
10 In Des "Poètes maudits" à "Tête de faune", Olivier BIVoRT porta la continuazione di una interessante ricerca, risultata quasi completamente infruttuosa per Michael Pakenham, sulla ricezione critica dei Poètes maudits. Oltre all'unico compte-rendu non repertoriato scovato da Pakenham nel «Voltaire», Bivort presenta infatti due altri articoli, apparsi nel 1884 in «Gil Blas» e nella "Revue critique». Se il primo è formato da una breve recensione anonima, il secondo, scritto da Charles Morice e ritrovato da Bivort alla biblioteca dell'Institut de France, contiene, oltre a una lunga ed entusiastica recensione, anche il testo inedito di Tête de faune di Rimbaud, posseduto da Verlaine ma non inserito nei Poètes maudits, e Brise marine di Mallarmé.

11 Rose BLIN-MIOCH (Louis-Xavier de Ricard et son "maitre" Edgard Quinet) rende qui omaggio a un altro autore caro a Pakenham, Louis-Xavier de Ricard, cofondatore del Parnasse. Seguendo la corrispondenza del poeta con Edgard Quinet, cui lo legano, oltre all'amicizia personale, la comune opposizione politica al secondo Impero e la collaborazione alla «Revue du progrès», l'A. propone i 1 testo - e un ampio commento di tre lettere inedite di Ricard à Quinet fra il 1874 e il 1875.

12 Rimbaud torna protagonista nel saggio di Eddie BREUIL "Les "Illuminations" de Rimbaud écrites à Londres en 1874» con l'annosa e a lungo controversa questione della datazione delle Illuminations e della loro organizzazione in recueil. Breuil ripercorre puntualmente gli avvenimenti e soprattutto gli scambi epistolari e di testi poetici fra Rimbaud e Verlaine - e poi con Nouveau - fra il 1873 e il 1874, riproponendo alcune delle considerazioni sui rapporti fra Rimbaud e Nouveau che l'hanno portato nel 2014 alle audaci tesi del volume Du Nouveau sur Rimbaud («Studi francesi» 176, pp. 397-398).

13 In Rimbaud «Carlisse»? Pierre BRUNEL approfondisce l'uso del termine «carlisme» fatto da Verlaine, già sottolineato da Pakenham, e il senso della deformazione verlainiana «carlisse» che compare in un dizain. Dopo una breve premessa sul significato del termine, legato alla triste sorte del don Carlos di Spagna ottocentesco e alla nascita del «parti carliste», Brunel passa ad esaminare i progetti più o meno velleitari di Verlaine e soprattutto di Rimbaud di un «engagement carliste», filtrati attraverso la corrispondenza con Nouveau e Delahaye.

14 Lo studio di Alain CHEVRIER, Jean Dayros et son double, è consacrato a un poeta poco noto, Paul Henry Colombier ossia Jean Dayros, autore della raccolta Les solitaires (1898). Entrando nel dibattito sull'attribuzione allo stesso autore di Mes États d'âme ou les Sept Chrysalides de l'extase, Chevrier compie un'accurata comparazione degli aspetti metrici e paratestuali delle due raccolte, mostrando la loro inequivocabile appartenenza «à la même plume».

15 L'analisi dettagliata della breve poesia mallarmeana «Soupir» è l'obiettivo del contributo di In-Ryeong CHOI, Sonate et perspective dans "Soupir" de Mallarmé, che vuole mostrare la nascita di una nuova poetica, precedente ad Hérodiade, fondata su una combinazione della forma solida di un volume con la «représentation des impressions fugitives». Sulle orme di Marc Dominicy, l'A. attribuisce il movimento del «soupir» alla «double mise en composition recourant à des procédés picturaux et musicaux: la perspective d'une part, la forme sonate de l'autre».

Bruno CLAISSE ritorna a Rimbaud con una celebre illumination: "Fleurs" de Rimbaud ou «la vie métaphorique». La triplice declinazione della metafora fleur/femme sullo sfondo sontuoso di una villa marina, «arrière-plan mondain» delle diverse metamorfosi, inizia con la digitale, continua con la rose d'eau e si conclude in crescendo con la foule des jeunes 
et fortes roses, tracciando la parabola «d'un temps perdu: celui d'une évasion fantasmatique aspirant à se substituer à la réalité».

Con la consueta precisione dei suoi studi metrico-stilistici, Benoît DE CORNULIER descrive e analizza «l'album de Roscoff» (Corbière pouëte précieux dans l'album de Roscoff?), insieme eterogeneo di testi poetici, in prosa e di disegni ritrovato nel 2010, in cui particolarmente significativa appare l'interazione fra poesia e disegno. La «poésieportrait de Rosalba», enigmatico insieme di versi tradizionali "d'une délirante précieusisté encombrée de clichés et d'archaïsmes, et sentant fort la parodie» è al centro dell'attenzione di de Cornulier, che ne sottolinea le continue contraddizioni, a un tempo stilistiche e sociali.

Un poète parnassien amateur d'art è l'omaggio di Joël DALANçon a Albert Mérat, poeta dimenticato e celebrato da Rimbaud come voyant, di cui l'A. ripropone qui «le souci de "peindre" en vers» attraverso tre testi poetici scritti a partire da tre opere pittoriche: «Le matin» per un quadro di Fromentin, «Sur une composition de F. Millet» e le tre quartine di «Pour le Polichinelle de Manet». Poeta malinconico e collezionista solitario, Mérat «laisse l'image d'un homme ombrageux, jaloux du succès des autres et peu inclin, dit Verlaine, aux "emballages"».

19 A Émile Le Brun, poco conosciuto amico di Verlaine, dedica uno studio Jean-Louis DEBAUVE, che raccoglie l'insieme delle dediche di Verlaine a Le Brun e delle note sparse su quattro esemplari di opere verlainiane da lui possedute. Le note, in parte attribuibili a Verlaine, riguardano Romances sans paroles, Sagesse (di cui Debauve riproduce le annotazioni non riportate nel saggio di Bernard Guégan), Jadis et Naguère con correzioni «à l'encre violette», e infine L'Amour, per il quale l'A. rinvia a un suo precedente studio.

In Enjeux esthétiques et politiques de "Qui veut des merveilles?", Solenn DUPAS prende in esame l'opera teatrale prodotta dalla collaborazione fra Verlaine e Coppée, Qui veut des merveilles, rivista satirica «où les plaisenteries inoffensives se mêlent à des allusions plus subversives», rappresentando un ampio quadro sociale in cui campeggiano $\mathrm{i}$ periodici del tempo e figure della modernità letteraria e dell'Accademia. Destinata ad essere letta più che messa in scena, la rivista esprime anche una specifica critica al regime di Napoleone III e alle recenti innovazioni tecnologiche.

21 Peter J. EDWARDS sposta l'attenzione su un'altra opera teatrale di Verlaine poco considerata, Les Uns et les autres, cui trova degli antenati illustri in Verlaine, Horace, Virgile et Théocrite. Ispirata all'ode oraziana «Donec gratus eram» e trasformata in «une pastorale des fêtes galantes» grazie all'ambientazione in un parco di Watteau, la pièce verlainiana rivela all'analisi dell'A. anche l'influenza di Teocrito e di Virgilio, che contribuiscono a farne, più che un'utopia, il malinconico «contre-idylle de la déception».

Louis FORESTIER presenta in À propos de Charles Cros, come precisa nel sottotitolo, un'interessante scoperta: «deux poèmes négligés et un inédit», i primi presso i librai Saunier e Lacroix, l'altro pubblicato in un oscuro periodico - «L'Écho bayeusain» - nel 1880. Nell'accurato esame dei due sonetti manoscritti, si conferma la passione di Cros per l'arte, qui quella «des maitres anciens» dato che non ha ancora incontrato gli impressionisti, e il ruolo centrale della figura femminile; il terzo testo, formato da quattro strofe di tredici versi e del tutto inedito, appare invece consacrato a un breve soggiorno ad Arromanches. 
23 Nel dibattito avviato da Fongaro e Brunel sulle fonti di Délires I di Rimbaud, rispettivamente riconosciute nella Sorcière di Michelet e nella parabola delle vergini del Vangelo secondo Matteo, Yann FRÉMY aggiunge qui diversi elementi (Les autres de Rimbaud), a partire dalle scene scolpite nel portale meridionale della cattedrale di Strasbourg, da cui Michelet ha tratto un'immagine di «Vierge folle» orgogliosamente sensuale, divenuta poi «la sorcière». «Il n'est donc pas exclu-afferma Frémy - que Rimbaud ait pu s'inspirer de l'élément plastique et pas seulement littéraire» pur sostenendo la sua cultura teologica e la possibile ispirazione anche da testi come lo Sponsus di Limoges e Il Cantico dei cantici. L'incertezza rimane: «Le lecteur de 1873 appréhendait-il semblable expression [«Vierge folle»] à travers le paradigme évangelique ou érotique?».

Sempre nel solco degli studi di Michael Pakenham, Michèle GORENC approfondisce in Jean Aicard et la poésie du pays natal un importante aspetto dell'opera di Jean Aicard, espresso nella raccolta Poèmes de Provence, in cui «l'universel et le local se complètent harmonieusement». La "poésie nouvelle» di Aicard, fondata su tre elementi - «choix thématique (le paysan)», "volonté de valoriser la province dans l'unité nationale» e infine scelta della lingua francese per esprimere il regionalismo letterario nell'unità della lingua nazionale -, sarà il modello di riferimento, dopo il 1880, di un'importante serie di raccolte poetiche e di racconti.

Le Spleen de Paris presenta per Jean-Michel GOUVARD (Baudelaire et la caricature) importanti analogie con l'arte della caricatura su diversi piani. L'A. ne illustra due esempi salienti - «Perte d'auréole» e «Le Joujou du pauvre»-, caratterizzati entrambi da un sintagma linguistico particolare: rispettivamente «la fange du macadam» e l'«égale blancheur». Illustrato da disegni di Daumier, Cham e Danjou, l'articolo mette in luce sia la profondità referenziale e simbolica delle caricature dell'epoca, sia la corrispondenza tematica con i poèmes en prose baudelairiani, cui sono legate anche dall'uso di alcuni «tropes visuels» trasposti da Baudelaire nei suoi testi.

Peter HAMBLY si ripropone, in Mallarmé, Banville et "Le Tombeau d'Edgar Poe", di rileggere la poesia mallarmeana alla luce dell'influenza di Banville e della corrispondenza fra il suo autore e Lefébure. La puntuale indicazione delle riprese banvilliane e l'incrocio con le osservazioni di Mallarmé nelle lettere («Devant le papier l'artiste se fait» della corrispondenza per il primo verso, e le numerose fonti banvilliane fra cui «Du sol et de l'éther hostiles, ô grief», «tailler l'image des Déesses/Dans le marbre», l'image du «granit» e molte altre) mostrano nel sonetto la preoccupazione costante di Mallarmé per «l'effet à produire», eredità profonda di Poe.

In Naissance d'une œuvre, Bénoît Houzé rivela ed esamina due manoscritti di Corbière, contenenti testi non inediti ma poco considerati-«Ay-Panneau» e la «Véritable complainte d'Auguste Berthelon» -, appartenenti alla raccolta di documenti posseduti dal cugino Jules-Édouard Chenantais, parodie rispettivamente della canzone «Ay Chiquita», celebre all'epoca, e della «Complainte de Fualdès». Houzé sottolinea fra l'uno e l'altro «un net infléchissement de la tonalité satirique»; all'oscenità dichiarata del primo si sostituisce «une sympathie pour Berthelon jusque dans ses ridicules» che riduce in parte l'ironia.

A uno scrittore/giornalista poco noto, collaboratore del quotidiano «La Justice» diretto da Clemenceau, è dedicato lo studio di Samuel LAIR, Sutter-Laumann, lecteur des "Amours jaunes" en 1887, studio che si fonda soprattutto sulla testimonianza di Gustave Geffroy, biografo di Clemenceau. È nella serie «Les poètes méconnus» che nel 1887 Sutter- 
Laumann pubblica «une recension contrastée de l'œuvre de Tristan, à la fois singulière dans sa fraîcheur, et ressortissant simultanément à l'influence de certaines idées reçues», singolarmente perspicace nell'indicare il corpo come primo punto di interesse del poeta.

Benché citato da Champfleury già nel 1852, Ange Pechméja, autore della raccolta poetica Strophes militantes, del romanzo Rosalie e del saggio L'œuf de Kneph, è approdato a un'antologia poetica solo all'inizio di questo secolo. Jean-Pierre LASSALLE mette in rilievo, in Ange Pechméja, linguiste et poète, i meriti linguistico/scientifici di questo poeta d'ispirazione esoterica che, malgrado alcuni limiti espressivi, annuncia secondo l'A. «la poésie "scientifique" voulue par André Chénier, puis, plus tard, par Sully Prudhomme».

Thérèse LASSALLE-MARAVAL prende in esame il momento della nascita della vocazione poetica di Valéry (1890-1893) a seguito della lettura di Mallarmé (De quelques images mallarméennes dans la poésie de Paul Valéry). In particolare vengono dettagliatamente studiate qui le variazioni introdotte da Valéry su alcune immagini fondamentali di Mallarmé - «l'Azur», «le Cygne», «les pierres ou métaux précieux» - attraverso le quali Valéry rivela anche il suo pensiero profondo sull'assurdità della condizione umana, che tuttavia non impedisce «de vivre de toutes les richesses de cette création».

31 Il titolo Quelques informations sur la découverte de l'“Album zutique" dans une lettre de Maurice Chalvet à Pascal Pia fornisce già tutte le principali coordinate della scoperta di Jean-Jacques LEFRÈRE e Jean-Paul GOUJON, che, dopo aver riprodotto la lettera inedita in questione (1962), tracciano dettagliatamente il percorso dell'Album, il cui manoscritto resta tuttora inaccessibile, transitato da Coquelin Cadet al «sieur de La Houssaye», a Chalvet, per arrivare infine - privato del ritratto di Rimbaud venduto a parte -, a Pascal Pia e alle sue edizioni del 1943 e del 1961.

Un altro punto controverso, l'incontro fra Rimbaud e Nouveau, è al centro dello studio di Cyril LHERMELIER e Seth WHIDDEN, Jean Richepin, une mémoire à trous? Passando attraverso numerose testimonianze e ricerche critiche, fra cui quella di Pakenham, i due autori fondano la loro ipotesi (Rimbaud e Nouveau si sarebbero incontrati, come afferma Delahaye, verso la fine del 1873 e avrebbero trascorso insieme una parte dell'inverno a Londra) sull'accurata disamina di tempi, luoghi e testimonianze, fornite soprattutto da alcune lettere di Raoul Ponchon, riprodotte anche in originale e confrontate con le dichiarazioni di Richepin nel suo articolo del 1927 sui due poeti, nonché con la lettera dell'aprile 1874 di Nouveau a Richepin.

33 A Laforgue, poeta caro a Pakenham, è dedicato il saggio di Rosemary LLOYD Laforgue et la création du soi. «Aucun écrivain du dix-neuvième siècle, pas même Baudelaire, - afferma l'A. - a su mieux que Laforgue employer un cliché pour en tirer une image nouvelle du soi» creando al tempo stesso una combinazione nuova di forme poetiche esistenti. $\grave{E}$ soprattutto nelle lettere (a Kahn, a Marie, a Charles Henry) e nell'abbozzo del suo romanzo autobiografico che appaiono le principali tracce di costruzione di una identità molteplice attraverso «une série de masques» e l'ascolto attento delle "présences étrangères qui jouent sur nous»; nel suo caso Baudelaire, Cros, Stendhal, Balzac, Taine, Hartmann...

Bertrand MARCHAL presenta in Petite chronique de la rue de Rome una serie di lettere scritte da Mme Mallarmé alla figlia Geneviève nel luglio 1893, in occasione del suo soggiorno a Honfleur presso Mme Ponsot. Un'accurata annotazione accompagna le otto lettere. 
Mary ORR, "Correspondances" de Baudelaire, si presenta come una rilettura «scientifica» del noto sonetto baudelairiano. «Scientifica» vuol dire per Orr non anacronistica, ma fondata sull'aspro dibattito del tempo «sur la place de l'homme (homo sapiens) dans l'ordre naturel des choses», come appare chiaro dalla prima quartina e dalla stratificazione delle epoche evocata dall'ambre, resina fossile estranea alla sequenza dei profumi. Eludendo la lettura cattolica come quella positivista, Baudelaire enuncia il caos senza risolverlo, se non nei «transports de l'esprit et des sens».

Michel PIERSSENS si occupa, nel suo studio Mirliton, sa vie, son œuvre, del diffondersi a partire dal 1880 delle «cartes postales» illustrate, di cui fornisce alcune riproduzioni, corredate da distici di vers de mirliton, esposti alla lettura di chiunque. Singole o in più sofisticate serie narrativamente progressive, le cartoline illustrate e i loro anonimi poeti sottolineano per Pierssens, «au tournant du siècle» il sentimento "d'une sinistre solitude», che si fa strada dietro l'esibizione massiccia di sentimenti preconfezionati.

Il testo baudelairiano di «Abel et Caïn» è l'oggetto della riflessione di Mario RICHTER in Forme et signification. È in effetti anzitutto l'organizzazione formale della poesia a suggerire il percorso di lettura-«l'unité dans la duplicité»-colto da Richter nell'alternanza dei distici, dedicati ad Abele e Caino, uniti tuttavia dall'intreccio dello schema metrico a rime alternate. La parola race, anaforicamente ripresa da tutti i distici, unisce ulteriormente il testo e trova conferma nell'interpretazione proposta da Richter per la doppia sineddoche «Le fer est vaincu par l'épieu», in cui la prevalenza è data alla comune sostanza che compone i due oggetti evocati. "En résumé, les deux 'races' qui devraient, bibliquement (culturellement) diviser les hommes [...] en bons et en méchants, etc., apparaissent, en réalité, comme une seule et même 'race'».

Studi Francesi, 183 (LXI | III) | 2017 

zutisme» già nelle opere del giovane Verlaine e particolarmente in quelle del 1867, accuratamente catalogate da Pakenham per quanto riguarda la produzione giornalistica. Anno di diffusione dei Poèmes saturniens appena pubblicati, il 1867 vede anche l'incontro con Hugo, i funerali di Baudelaire e l'inizio della produzione satirica di Verlaine nella rubrica Nos murailles littéraires («France artistique») e produzioni satiriche di gruppo come la Complainte de Berezowski, che prefigurano già in parte il «cercle zutique» che nascerà nel 1871, «peut-être encore aujourd'hui la seule barricade de la Commune encore debout et la première barricade poético-politique de l'Histoire». caratterizzano la poesia della modernità - da Baudelaire a Rimbaud, Lautréamont, Cros, Laforgue et Corbière - Denis SAINT-AMAND privilegia (D'hideusement beaux rossignols de la boue) la funzione fondamentale dell'ossimoro, «forme d'abouchement de l'opposition au sein d'un seul syntagme». Attraverso alcuni testi di poesia e prosa baudelairiani, l'A. mostra la centralità del procedimento, che ricompare vistosamente nell'opera di Rimbaud fin dai primi componimenti («Vénus Anadyomène») e poi nelle Illuminations, nei Chants de Maldoror, nei testi di Cros e di Corbière, segno di una convivenza non risolta, a partire da Baudelaire, «entre le romantisme qu'il voit mourir et une poésie moderne en marche».

«Il est malaisé de mesurer avec exactitude l'ampleur [...] des échos verlainiens et leur persistence dans les textes de Laforgue» afferma Henri SCEPI all'inizio di Laforgue lecteur de Verlaine, in cui documenta e approfondisce le «modalités d'un dialogue» fra i due poeti. Negli anni dal 1880 al 1885, in cui si forma il progetto delle Complaintes, la scena poetica appare in preda a tensioni contrastanti - la parodia delle Déliquescences corrode i valori ancora imperanti della scuola parnassiana -, ma il personaggio di Verlaine aleggia dappertutto con la sua «poétique de l'imprécis». Ma in che cosa consiste «la manière Verlaine»? Cercando di fornire una risposta, Scepi indica anzitutto la lingua, «parole volontairement réduite à son substrat oral», poi «une esthétique de l'immédiat» articolata sulla "naïveté» della sensazione: è a questa poetica della semplicità che Laforgue aderisce, aggiungendovi la sua «petite névrose», la sua filosofia della rinuncia e dell'inconscio, sostenuta anche dalle teorie di Hartmann.

A Leah Laforgue, moglie dell'autore delle Complaintes, è consacrato l'articolo di Martin SORRELL, in risposta a una suggestione di ricerca datagli dello stesso Michael Pakenham nel 1970. Di Leah Lee, che Laforgue chiamava «petit personnage», Sorrell presenta un ritratto dettagliato e a tratti affettuoso, necessariamente breve come la tragica vita di Jules e Leah, uniti da un brevissimo matrimonio e dalla morte sopraggiunta per entrambi a 27 anni.

Con Les chiffonniers de Stéphane Mallarmé Michael TILBY porta l'attenzione su «Galanterie macabre», (1861), conosciuta solo nel 1930. L'immagine dei «chiffonniers», molto diffusa e di evidente origine baudelairiana, appare decisamente banale nella letteratura dopo il 1850, ma, come mostra Tilby attraverso un'accurata analisi del testo, Mallarmé non appare realmente interessato ad essa, per la ragione che il protagonista reale della poesia è «le poète-chiffonnier», che raccoglie come oggetti abbandonati gli echi dispersi di altri testi poetici. Di qui, per Tilby, la distanziazione dal tono celebrativo di «Le Vin des chiffonniers», fonte comunemente riconosciuta, e una più significativa vicinanza ai Petits poèmes en prose: come Baudelaire prosatore, Mallarmé presenta qui «une combinaison provocatrice d'un banal récit» e del modo in cui esso «stimule 
l'imagination du poète à cumuler des comparaisons ironiques et pertinentes sur le plan universel des choses».

Le "Sonnet des sept nombres", ou comment être poète au piano. Ispirato dallo studio di Lefrère e Pakenham, Cabaner, poète au piano, Charles-Étienne tREMBLAY mette in prospettiva la lettura del Sonnet des sept nombres di Cabaner «en tant qu'œuvre de pianiste, d'une part, et de parodiste des Voyelles, d'autre part». Fondata su «un souci de logique», l'opera di Cabaner, di cui Tremblay analizza qui le teorizzazioni musicali espresse nel manoscritto del sonetto da piccoli indizi sparsi, tende così a supplire ai difetti espressivi del pianoforte e ad arrivare «à une pensée chantée et comprise du chanteur»: «le texte de Cabaner contiendrait donc son accompagnement» e renderebbe così l'opera completa ed «explicite», cioè realmente comunicativa.

Jean-Didier WAGNeUR (Publier après la Commune) propone nel suo articolo una serie di riflessioni sulle lettere inviate da Léon Cladel, romanziere e direttore de «La Marseillaise» a Arthur Arnould fra il 1878 e il 1880. Scrittore e giornalista rivoluzionario, Arnould, membro attivo della Comune rifugiatosi poi a Ginevra, appare come il destinatario di una corrispondenza centrata sulla cultura del tempo e sul difficile rapporto degli scrittori con la società e il potere politico della Terza Repubblica, mentre cerca a sua volta di reinserirsi, anche attraverso Cladel, nella «Société des gens de lettres».

Éric WALBECQ presenta in «Mme Nina de Callias, une farceuse artistique» il "dossier de surveillance de la préfecture» del 1872 al 1876, relativo a Nina de Villard, autrice molto amata da Michael Pakenham, arricchito dalla riproduzione di un probabile ritratto della poetessa eseguito da Charles de Sivry. Mai pubblicato per intero, il dossier fornisce interessanti osservazioni su Nina e sugli autori a lei vicini, come Cros, Richepin, Mendès e altri.

Ray Davison conclude il volume con un omaggio affettuoso ai vari aspetti della ricca personalità di Pakenham: «le bon viveur», «le connoisseur», «le bricoleur», «the gymnaste», «the rebel», «the scholar», «the balzacien». 